\title{
Magnetic Structure, Phase Diagram, and a New Type of Spin-Flop Transition Dominated by Higher Order Interaction in a Localized $5 f$ System $\mathbf{U}_{3} \mathbf{P d}_{20} \mathbf{S i}_{6}$
}

\author{
Y. Koike, ${ }^{1,2}$ N. Metoki, ${ }^{1}$ Y. Haga, ${ }^{1}$ K. A. McEwen, ${ }^{3}$ M. Kohgi, ${ }^{4}$ R. Yamamoto, ${ }^{4}$ N. Aso,${ }^{5}$ N. Tateiwa, ${ }^{6}$ T. Komatsubara, \\ N. Kimura, ${ }^{7}$ and H. Aoki ${ }^{7}$ \\ ${ }^{1}$ Advanced Science Research Center, Japan Atomic Energy Research Institute, Tokai, Ibaraki, 319-1195, Japan \\ 2 Japan Science and Technology Corporation, Kawaguchi, Saitama 332-0012, Japan \\ ${ }^{3}$ Department of Physics and Astronomy, University College London, Gower Street, London WC1E 6BT, United Kingdom \\ ${ }^{4}$ Department of Physics, Tokyo Metropolitan University, Hachioji, Tokyo 192-0397, Japan \\ ${ }^{5}$ Neutron Scattering Laboratory, Institute for Solid State Physics, University of Tokyo, Ibaraki 319-1106, Japan \\ ${ }^{6}$ Research Center for Materials Science at Extreme Conditions, Osaka University, Toyonaka, Osaka 560-8531, Japan \\ ${ }^{7}$ Center for Very Low Temperature Science, Tohoku University, Sendai 980-8545, Japan
}

(Received 28 December 2001; published 26 July 2002)

\begin{abstract}
The magnetic structure of the localized-5f uranium intermetallic compound $\mathrm{U}_{3} \mathrm{Pd}_{20} \mathrm{Si}_{6}$ has been determined by means of a neutron diffraction experiment. Our data demonstrate that this compound has a collinear coupling of the sublattice ordering of the uranium spins on the $4 a$ and $8 c$ sites. We conclude that higher-order exchange and/or quadrupole interactions are necessary to stabilize this unique collinear structure. We discovered a new type of spin-flop transition against the uniaxial anisotropy induced by this collinear coupling.
\end{abstract}

DOI: 10.1103/PhysRevLett.89.077202

PACS numbers: 75.30.-m, 61.12.Ld

Actinide-based intermetallic compound is one of the most attractive systems in the field of strongly correlated electron systems [1]. The main difficulty is how to treat the renormalized quasiparticles, dominating the ground-state properties. Spin and quadrupole degree of freedom with strong Hund coupling should be taken into account for the many-body effect, where the configuration of $5 f$ electrons is very important. However, the configuration in most uranium intermetallic compounds is unknown. Recently, we found a new system, $\mathrm{U}_{3} \mathrm{Pd}_{20} \mathrm{Si}_{6}[2,3]$. The observation of clear crystalline electric field excitations [4,5] provides direct evidence of the localized nature with the $5 f^{2}\left(\mathrm{U}^{4+}\right)-\Gamma_{5}$ triplet ground state $[2,3,5]$.

In addition to the $5 f$ configuration, $\mathrm{U}_{3} \mathrm{Pd}_{20} \mathrm{Si}_{6}$ presents a good opportunity to study a magnetic order which is frustrated due to the cancellation of the Heisenberg interaction between two magnetic sublattices. Our recent neutron scattering study revealed the existence of the successive ordering; uranium spins on the simple cubic $8 c$ lattice order antiferromagnetically below $T_{\mathrm{N}}=19 \mathrm{~K}$, while the fcc- $4 a$ sites show ferromagnetic ordering below $T_{\mathrm{C}}=2 \mathrm{~K}$ [4]. Since the ground-state magnetic structure is unknown, it is unclear whether (and how) the frustration could be released. The origin of the metamagnetic transition also remains an open question [6].

In this paper, we report the discovery of a remarkable collinear magnetic structure stabilized by higher-order coupling of an antiferromagnetic $8 c$ lattice to an interpenetrating ferromagnetic $4 a$ lattice. This structure shows a new type of field-induced spin flop caused by the uniaxial anisotropy due to the collinear coupling. The ferromagnetic moment is strongly suppressed by the intersite coupling. The magnetic moment recovers its saturation moment under a magnetic field. The moment size guarantees that this compound is a prototype of the localized $5 f$ magnet with $5 f^{2}-\Gamma_{5}$ ground state.

Neutron scattering experiments were carried out on the triple-axis-spectrometer TAS-2 $\left(E_{i}=13.7 \mathrm{meV}\right)$ at the research reactor JRR-3 in the Japan Atomic Energy Research Institute. A single crystal $(0.5 \mathrm{~mm}$ thick and $5 \mathrm{~mm}$ in diameter) was mounted with the [100] and [011] axes in the horizontal scattering plane. A magnetic field was applied either along the vertical, $\boldsymbol{H}_{\perp} \|[0 \overline{1} 1]$, or horizontal direction, $\boldsymbol{H}_{\|} \| \boldsymbol{Q}$.

Figure 1 summarizes the $H-T$ phase diagram for the magnetic structures of $\mathrm{U}_{3} \mathrm{Pd}_{20} \mathrm{Si}_{6}$ determined in this study. There are three ordered phases. In antiferromagnetic(AF)phase uranium spins on the $8 c$ sites order in the G-type antiferromagnetic structure. The magnetic moment is parallel to $\langle 100\rangle$. In the antiferromagnetic + ferromagnetic $(\mathrm{AF}+\mathrm{FM})$-phase, the ferromagnetic ordering on the $4 a$ sites shows a collinear coupling with $8 c$ antiferromagnetic ordering. The $8 c$ spins exhibit spin-flop (SF) transition for $H \sim 5 \mathrm{~T}$, and the SF-phase becomes stable for higher magnetic fields.

In AF-phase, magnetic Bragg peaks were observed at odd integer reflections, indicating the antiferromagnetic ordering on $8 c$ sites with the propagation vector $\boldsymbol{Q}=$ $\langle 111\rangle$ [4]. From the lattice symmetry, multi- $\boldsymbol{Q}$ structures can be ruled out. The magnetic structure factor $F(h k l)$ is given by

$$
F(h k l)=8 \mu_{8 c}^{\mathrm{AF}} f(Q) \sin \left(\nu_{8 c}\right) \quad(h, k, l: \text { Odd integers }),
$$

where $\mu_{8 c}^{\mathrm{AF}}, f(Q)$, and $\sin \left(\nu_{8 c}\right)$ are the moment size on the 


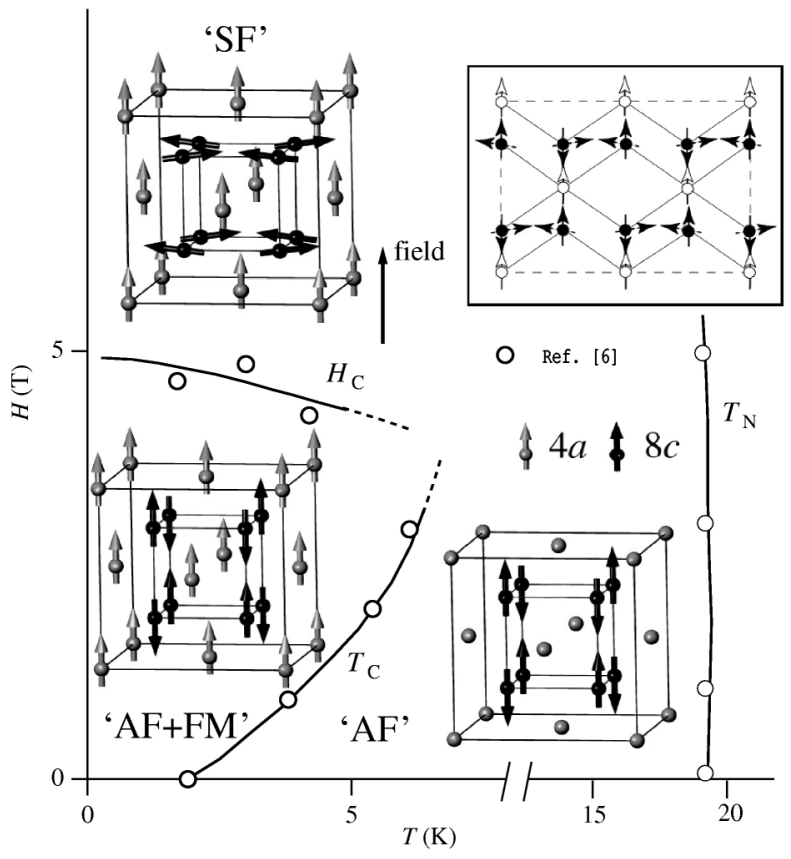

FIG. 1. $H$-T phase diagram [6] and the magnetic structure in $\mathrm{U}_{3} \mathrm{Pd}_{20} \mathrm{Si}_{6}$. Inset shows the projection of the uranium atoms on the (110) plane.

$8 c$ sites, magnetic form factor [7], and the angle factor, respectively. As shown in Fig. 2 our data are well explained with Eq. (1), assuming $\mu_{8 c}^{\mathrm{AF}}=(1.83 \pm 0.05) \mu_{\mathrm{B}}$ and averaged angle factor $\left\langle\sin ^{2}\left(\nu_{4 a}\right)\right\rangle=2 / 3$ in a cubic system. This result is in good agreement with a recent powder diffraction study [4], and is consistent with the $\Gamma_{5}$ ground state, $2 \mu_{\mathrm{B}}$.

When $4 a$-FM and $8 c$-AFM ordering coexist below $T_{\mathrm{C}}=$ $2 \mathrm{~K}, F(h k l)$ can be described as the sum of both contributions: For odd integer reflections,

$$
\begin{aligned}
& F(h k l)=4 \mu_{4 a} f(Q) \sin \left(\nu_{4 a}\right)+8 i \mu_{8 c}^{\mathrm{AF}} f(Q) \sin \left(\nu_{8 c}^{\mathrm{AF}}\right) \\
& (h, k, l: \text { Odd integers }),
\end{aligned}
$$

and for even integer reflections,

$$
\begin{aligned}
& F(h k l)=4 \mu_{4 a} f(Q) \sin \left(\nu_{4 a}\right)+8 \mu_{8 c}^{\mathrm{FM}} f(Q) \sin \left(\nu_{8 c}^{\mathrm{FM}}\right) \\
& (h+k+l=4 n, n: \text { integers }), \\
& F(h k l)=4 \mu_{4 a} f(Q) \sin \left(\nu_{4 a}\right)-8 \mu_{8 c}^{\mathrm{FM}} f(Q) \sin \left(\nu_{8 c}^{\mathrm{FM}}\right) \\
& (h+k+l=4 n+2, n \text { : integers }),
\end{aligned}
$$

where $\mu_{4 a}$ denotes the magnetic moment on the $4 a$ sites, $\sin \left(\nu_{4 a}\right)$ is the angle factor, and $\mu_{8 c}^{\mathrm{FM}}$ is the ferromagnetic component of the $8 c$ sites. We found that the observed intensities for $T=0.3 \mathrm{~K}$ and $H=0 \mathrm{~T}$ in the (AF + FM)-phase can be explained with Eqs. (2)-(4), assuming $\mu_{4 a}=(1.1 \pm 0.3) \mu_{\mathrm{B}}, \mu_{8 c}^{\mathrm{FM}}=0$, and $\left\langle\sin ^{2}\left(\nu_{4 a}\right)\right\rangle=2 / 3$. It should be noted that the relative orientation of the $4 a$ and $8 c$ magnetic moments could not be determined with the measurements on a multidomain sample. No significant

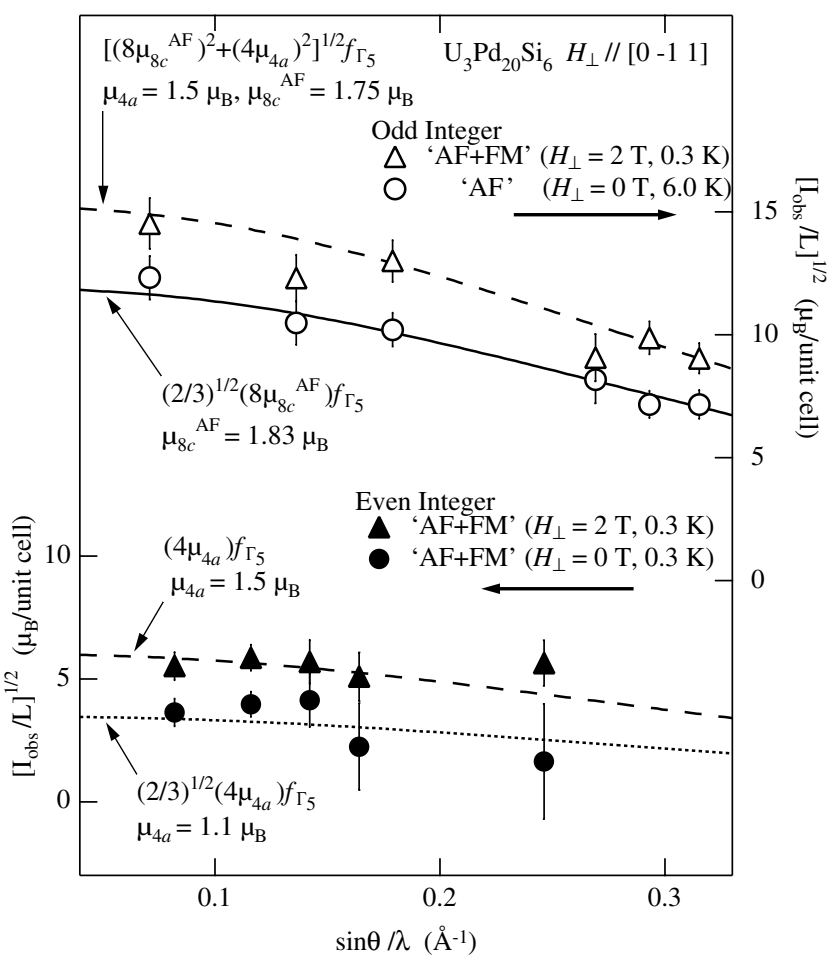

FIG. 2. Square root of the integrated intensities of the magnetic scattering divided by the Lorentz factor. The lines are calculated results. See text.

change was observed in $\mu_{8 c}^{\mathrm{AF}}$ below and above $T_{\mathrm{C}} \cdot \mu_{4 a}$ is considerably suppressed compared to the expected value $2 \mu_{\mathrm{B}}$ in the $\Gamma_{5}$ ground state. We will see later that $\mu_{4 a}$ recovers under an external field.

The existence of the collinear structure was confirmed by means of a neutron diffraction study under a magnetic field. The (022) and (222) ferromagnetic intensities in the $(\mathrm{AF}+\mathrm{FM})$-phase almost vanish with the application of a horizontal field $\left(\boldsymbol{H}_{\|} \| \boldsymbol{Q}\right)$ and the (022) intensity increases with the application of a vertical field $\boldsymbol{H}_{\perp}$ as shown in Fig. 3. These data indicate that the ferromagnetic domains merge into a single domain. A similar field dependence was observed for the (111) antiferromagnetic intensity in $(\mathrm{AF}+\mathrm{FM}$ )-phase (solid circles in Fig. 3), while no significant change was observed in AF-phase (open circles). This indicates that the $8 c$ spins change direction with collinearly coupled $4 a$ spins. The lack of change of the (111) intensity in AF-phase can be reasonably understood in terms of the domain structure and the angle factor.

The ground-state collinear structure is direct evidence of the collinear interaction between $4 a$ and $8 c$ spins. If the collinear interaction is negligibly weak, the SF-phase should be stable for $H=0$, because of the molecular field on the $8 c$ sites from the ferromagnetically ordered $4 a$ sites.

This collinear interaction and weak anisotropy enable us to form a single domain (AF + FM)-phase with application of a field. As shown in Fig. 2, the observed magnetic intensity at $H_{\perp}=2 \mathrm{~T}$ can be explained by assuming the 


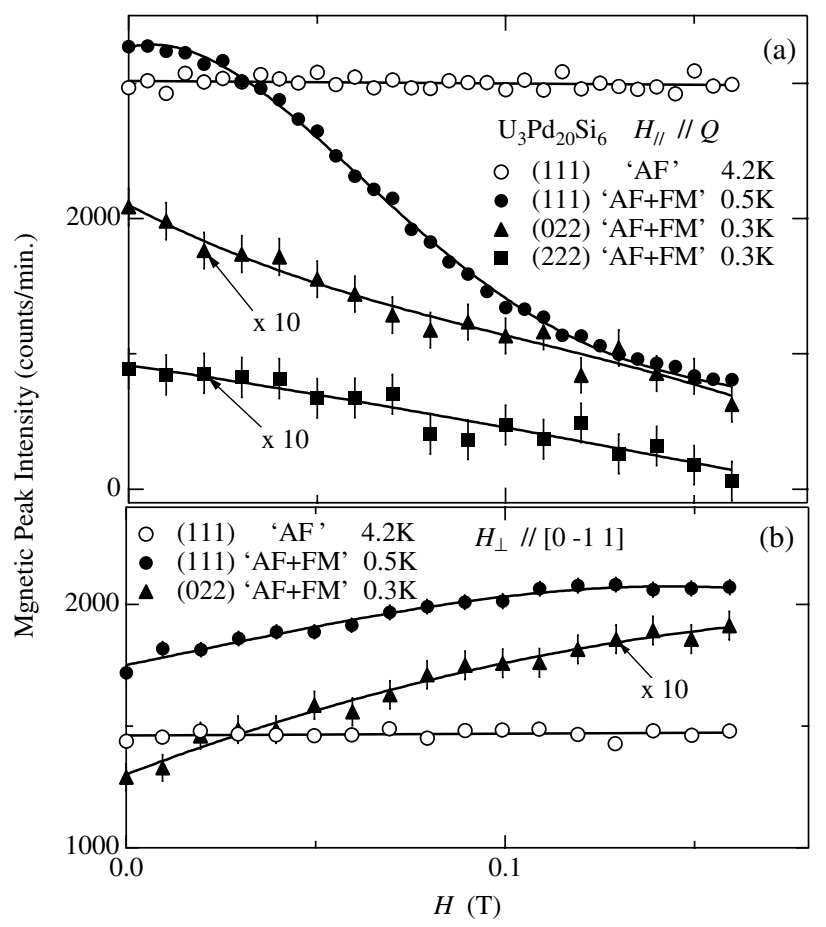

FIG. 3. $H_{\|}$(a) and $H_{\perp}$ (b) dependence of the magnetic peak intensity at (111), (022), and (222) Bragg points. The lines are guides for the eyes.

collinear structure with $\mu_{4 a}=(1.51 \pm 0.06) \mu_{\mathrm{B}}, \mu_{8 c}^{\mathrm{AF}}=$ $(1.75 \pm 0.03) \mu_{\mathrm{B}}$, and $\mu_{8 c}^{\mathrm{FM}}=0$, where the angle factors are unity.

Figure 4 shows the vertical field dependence of the magnetic intensity. The intensity at (111) exhibited a steep decrease from $H_{\perp}=3$ to $6 \mathrm{~T}$. We also observed an abrupt increase at the (022) and decrease at the (200) ferromag-

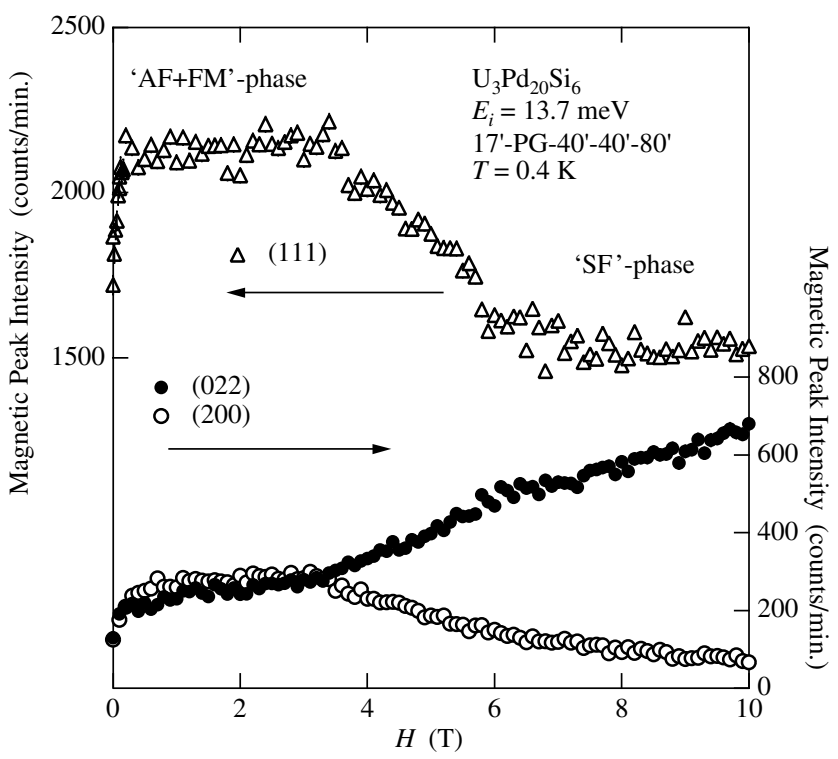

FIG. 4. $\quad H_{\perp}$ dependence of the (111), (200), and (022) magnetic peak intensities at $T=0.4 \mathrm{~K}$. netic Bragg peaks in the same field region. This intensity change indicates the spin-flop transition of $8 c$ spins.

Figure 5 summarizes the integrated intensities of the magnetic reflections in the SF-phase under $H_{\perp}=10 \mathrm{~T}$ at $T=0.3 \mathrm{~K}$. The observed intensities at odd integer reflections can be satisfactorily explained, assuming that the antiferromagnetic component of the $8 c$ spin $\mu_{8 c}^{\mathrm{AF}}=$ $(1.65 \pm 0.09) \mu_{\mathrm{B}}$ is parallel to the [100] axis in the scattering plane and the $4 a$ ferromagnetic component $\mu_{4 a}=$ $(1.64 \pm 0.09) \mu_{\mathrm{B}}$. The experimental result disagrees with calculated intensities in the case that the $8 c$ spins are parallel to the other high symmetry axes, [011] and [111] (or $[1 \overline{1} \overline{1}]$ ), which are also in the scattering plane. This means that the magnetic easy axis of the $8 c$ spins is parallel to the principal axis 100 .

A ferromagnetic component of the $8 c$ spins, $\mu_{8 c}^{\mathrm{FM}}=$ $0.41 \mu_{\mathrm{B}}$, was necessary to understand the ferromagnetic Bragg intensities. The canting angle of the $8 c$ spins was estimated to be approximately $15^{\circ}$. The moment $\mu_{4 a}=$ $1.64 \mu_{\mathrm{B}}$ was strongly enhanced with field application.

The field $\left(H_{\perp}\right)$ dependence of the magnetic moment is shown in Fig. 6. The ferromagnetic component of the $8 c$ sites exhibits a steep increase in the vicinity of the spin-flop field. $\mu_{4 a}$ recovers the value in the $\Gamma_{5}$ ground state. The total moment per uranium atom $\left(\mu_{4 a}+2 \mu_{8 c}^{\mathrm{FM}}\right) / 3$ obtained by the neutron diffraction study is in perfect agreement with the result of a recent magnetization measurement [6].

The spin-flop transition is unusual, because (i) it is not expected with cubic anisotropy and (ii) the spin flop of the

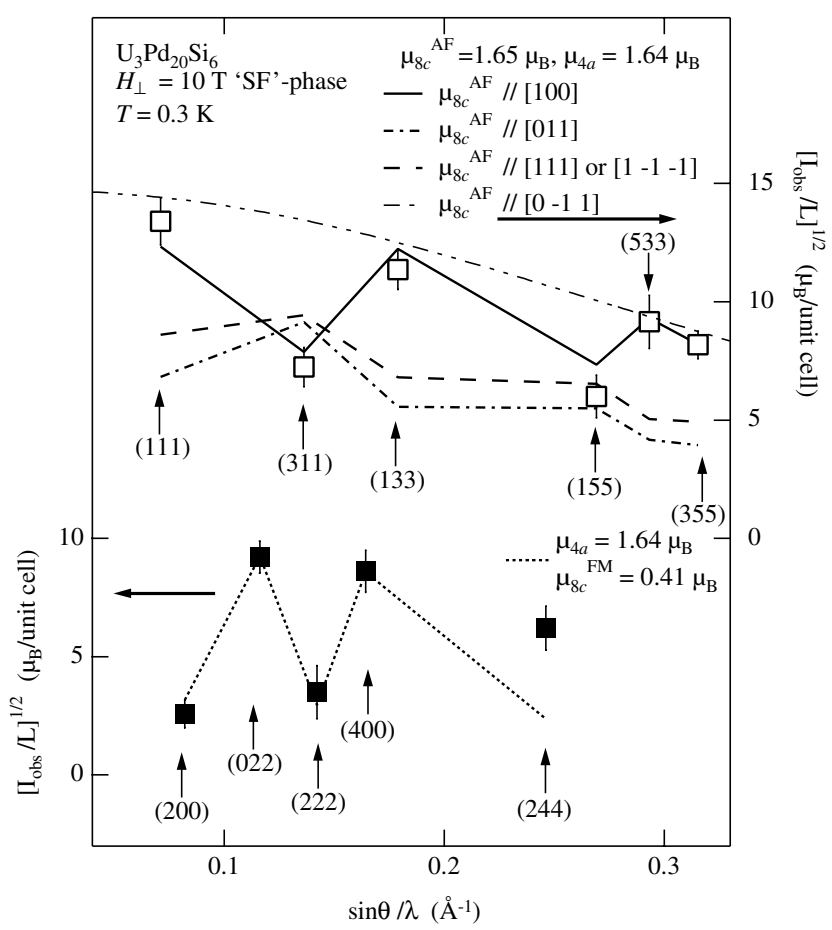

FIG. 5. Integrated intensity of the magnetic scattering for $H_{\perp}=10 \mathrm{~T}$ and $T=0.3 \mathrm{~K}$. 


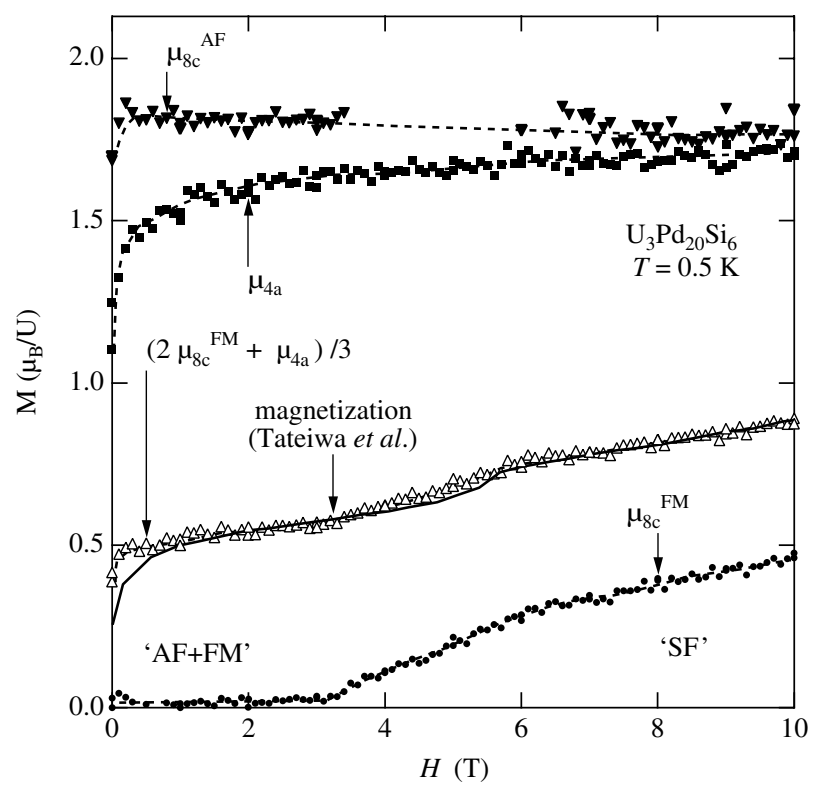

FIG. 6. $H_{\perp}$ dependence of $\mu_{8 c}^{\mathrm{AF}}, \mu_{8 c}^{\mathrm{FM}}$, and $\mu_{4 a}$. The broken lines are a visual guide. The total moment $\left(\mu_{4 a}+2 \mu_{8 c}^{\mathrm{FM}}\right) / 3$ is also plotted and compared with the result of the dcmagnetization measurement [6] denoted by a solid line.

$8 c$ sites is observed, when both $4 a$ and $8 c$ spins are ordered. We concluded that it is a new type of spin-flop transition against the collinear interaction which induces uniaxial anisotropy on the $8 c$ sites.

We infer that the collinear coupling is due to higherorder interaction. Heisenberg exchange interaction between $4 a$ and $8 c$ sublattices is canceled, and this is the reason why the $4 a$ and $8 c$ spins order at different temperatures. The transition temperatures, $T_{\mathrm{C}}=2 \mathrm{~K}$ and $T_{\mathrm{N}}=$ $19 \mathrm{~K}$, indicate the strength of the intrasite interactions. The cancellation of the dipole field is also apparent from the crystal structure.

We propose two possibilities for the origin of the collinear interaction, spin, and quadrupole channels. The first possibility is the higher-order exchange interaction,

$$
\begin{aligned}
\mathcal{H}= & -K_{1} \sum\left(S_{i} S_{j}\right)^{2} \\
+ & K_{2} \sum\left[\left(S_{i} S_{j}\right)\left(S_{k} S_{l}\right)+\left(S_{l} S_{i}\right)\left(S_{j} S_{k}\right)\right. \\
& \left.-\left(S_{k} S_{i}\right)\left(S_{j} S_{l}\right)\right] .
\end{aligned}
$$

In Eq. (5) the first and second terms describe biquadratic and 4-spin exchange interactions, respectively. The fourthorder perturbation gives a positive sign for $K_{1}$; hence a collinear structure is energetically favorable. When four spins $s_{i}(i=1$ to 4$)$ are all parallel to one plane, the 4-spin exchange interaction takes an extremum value, and in this case it can be expressed as

$$
K_{2} \cos \left(\phi_{1}-\phi_{2}+\phi_{3}-\phi_{4}\right)
$$

where $\phi_{i}$ is an angle of $s_{i}$ measured from a certain axis in this plane, and $K_{2}$ is positive [8]. In the crystal structure of $\mathrm{U}_{3} \mathrm{Pd}_{20} \mathrm{Si}_{6}$, there is a diamond network of nearest-neighbor spins as shown in the inset of Fig. 1. Equation (6) stabilizes the collinear structure, while the SF-phase is unstable for $H=0$.

The second possibility is the quadrupole interaction. The $\Gamma_{5}$ triplet ground state has the quadrupolar degree of freedom as demonstrated by the softening of $c_{44}$ [2]. The quadrupolar Hamiltonian $\mathcal{H}_{Q}=-\sum K_{\Gamma i j} O_{\Gamma i} O_{\Gamma j}$ can be described by the sum of the fourth-order terms of the $\boldsymbol{J}$ operator [9]. A biquadratic term which stabilizes the collinear coupling could be involved.

The strong suppression of the $4 a$ ferromagnetic moment could be explained by the intersite coupling. Below $T_{\mathrm{C}}$, a constant molecular field on the $8 c$ sites is expected from the $4 a$ sites. This molecular field might perturb the antiferromagnetic structure of $8 c$ spins. Therefore, the $4 a$ ferromagnetic ordering could be supporessed so as not to disturb $8 c$ antiferromagnetic ordering. In this situation, the magnetic moment would recover to the saturation value with field application.

In summary, the magnetic structure and $H-T$ phase diagram of a localized $5 f$ system $\mathrm{U}_{3} \mathrm{Pd}_{20} \mathrm{Si}_{6}$ have been studied by means of a neutron diffraction experiment. We found the existence of higher-order collinear coupling and a new type of metamagnetic transition against this collinear coupling.

We thank M. Date, M. Tachiki, K. Ueda, and G.H. Lander for stimulating discussions.

[1] See, e.g., V. Sechovsky and L. Havela, Handbook of Magnetic Materials, edited by K.H.J. Buschow (Elsevier Science, Amsterdam, 1998), Vol. 11, p. 1.

[2] N. Tateiwa, Ph.D. thesis, Tohoku University, 1998.

[3] N. Tateiwa et al., J. Phys. Soc. Jpn. 69, 1517 (2000).

[4] N. Tateiwa et al., J. Phys. Soc. Jpn. 70, 2425 (2001).

[5] K. Kuwahara et al., Physica (Amsterdam) 312-313B, 899 (2002).

[6] N. Tateiwa et al., J. Phys. Soc. Jpn. 70, 1853 (2001).

[7] A. J. Freeman et al., Phys. Rev. B 13, 1168 (1976).

[8] K. Yoshida et al., J. Phys. Soc. Jpn. 50, 3268 (1981).

[9] P. Thalmeier and B. Lüthi, in Handbook on the Physics and Chemistry of Rare Earths, edited by K. A. Gschneidner, Jr. and L. Eyring (Elsevier Science B.V., Amsterdam, 1991), Vol. 14, p. 225. 